\title{
Estimating Breathing Movements of the Chest and Abdominal Wall Using a Simple, Newly Developed Breathing Movement-Measuring Device
}

\author{
Hideo Kaneko PhD
}

\begin{abstract}
BACKGROUND: The 3-dimensional (3D) distances of specific points on the chest and abdominal wall are useful for assessing the characteristics of breathing movement. This study aimed to determine whether the 3D distances on the chest and abdominal wall during deep breathing (DB) could be estimated using a newly developed breathing movement-measuring device (BMMD). METHODS: Breathing movement distances on the chest (bilateral third and eighth ribs) and abdomen (upper abdomen) of $\mathbf{1 0}$ healthy young males were measured using a pen-sized mechanical BMMD and a 3D motion analysis system during DB. DB was controlled and based on the distances targeted by the BMMD (ie, 1, 2, and $3 \mathrm{~cm}$ ), which was placed on the observation points and fixed on a tripod. Simultaneously, the 3D distances of reflective markers on the observation points were measured. The obtained data were statistically analyzed using a paired $t$ test, repeated measures analysis of variance with multiple comparisons tests, and linear regression with mixed effects. RESULTS: All the BMMD distances were less than the 3D distances. The differences between the 3D and BMMD distances on the chest and abdomen, except for the left lower chest (eighth rib), were significantly greater at a target distance of $3 \mathrm{~cm}$ than that of $1 \mathrm{~cm}$. The coefficients of determination ranged from 0.98 to 0.99 . CONCLUSIONS: Linear regression equations provided adequate estimates of the 3D distances of markers on the chest and abdominal wall during DB. The results suggest that this BMMD is useful for quantitatively assessing DB movement of the chest and abdominal wall in healthy young males. Key words: breathing; breathing movement; chest wall; abdominal wall; 3-dimensional motion analysis; device. [Respir Care 2014;59(7):1133-1139. (C) 2014 Daedalus Enterprises]
\end{abstract}

\section{Introduction}

Chest and abdominal wall mobility is an important component of respiratory assessment. Loss of chest and abdominal wall mobility affects respiratory function and, in

\footnotetext{
Department of Physical Therapy, School of Health Sciences at Fukuoka, International University of Health and Welfare, Fukuoka, Japan

This research was supported by Japan Society for the Promotion of Science grant-in-aid for scientific research 22500472. The author has disclosed no other conflicts of interest.

Correspondence: Hideo Kaneko PhD, Department of Physical Therapy, School of Health Sciences at Fukuoka, International University of Health and Welfare, 137-1 Enokizu, Okawa-shi, Fukuoka 831-8501, Japan. Email: hkaneko@iuhw.ac.jp.
}

DOI: $10.4187 /$ respcare. 02778 some cases, results in respiratory complications. A loss in mobility may be due to various factors such as aging, ${ }^{1,2}$ scoliosis, ${ }^{3,4}$ thoracoabdominal surgery, ${ }^{5,6}$ ankylosing spondylitis, ${ }^{7}$ and other chest wall diseases. ${ }^{8}$ Therefore, chest and abdominal mobility assessments should be performed in a variety of clinical settings.

To accurately measure chest and abdominal wall movements, several noninvasive methods are available, including magnetometers, ${ }^{9}$ respiratory inductive plethysmography, ${ }^{10}$ optoelectronic plethysmography, ${ }^{11}$ and a respiratory movement-measuring instrument. ${ }^{12}$ However, these methods are rarely used in clinical practice because they are expensive and require well-trained technicians. Thus, to assess chest wall mobility, chest excursion has been measured using a tape measure. ${ }^{13,14}$ Although tape measurements are simple and inexpensive, this method cannot be used to evaluate any asymmetry or abnormality in chest and abdominal wall mobility, because the measured values 
reflect changes in chest and abdominal circumference, and the reference values used have not been definitively shown to be useful for judging abnormalities.

In a previous study, ${ }^{2}$ we attempted to establish reference values and to determine the characteristics of chest and abdominal wall movements during breathing for objective assessments during physical examinations. For this purpose, we used a 3-dimensional (3D) motion analysis system to measure the 3D breathing movement distances (at the inspiratory phase of the respiratory cycle) of the chest and abdomen of healthy subjects 20-74 y of age. This 3D motion analysis system, a so-called optical device, allows accurate assessments of the kinematics of the chest and abdominal wall. Optical devices for measuring motion require only lightweight reflective markers that create minimum interference in the measuring system when used with subjects and can be placed on any region(s) of interest. The results of our previous study ${ }^{2}$ showed that the $3 \mathrm{D}$ distances of observation points may be useful for assessing breathing movements during physical examinations, because they are related to the effects of age, gender, and posture, as has also been shown in previous studies.

For a more objective assessment of 3D distances of observation points in clinical practice, a breathing movement-measuring device (BMMD) was developed as a simple device that could be used anywhere. A previous study showed that this BMMD was useful for quantitatively assessing chest and abdominal wall movements during quiet breathing (QB). ${ }^{15}$ However, the accuracy of this BMMD for assessing chest and abdominal wall mobility during deep breathing (DB) has not been investigated. Thus, the purpose of this study was to determine whether the breathing movement distances of the chest and abdominal wall were adequately estimated using this BMMD in healthy young males.

\section{Methods}

\section{Subjects}

This study was performed at the School of Rehabilitation Sciences at Fukuoka, International University of Health and Welfare, Fukuoka, Japan. Ten healthy young males participated in this study. Subjects were recruited from students of the School of Rehabilitation Sciences at Fukuoka, International University of Health and Welfare. They had a mean age of $21 \pm 1$ (SD) y, mean height of $1.71 \pm .08 \mathrm{~m}$, mean weight of $64 \pm 10 \mathrm{~kg}$, and mean body mass index of $21.8 \pm 2.0 \mathrm{~kg} / \mathrm{m}^{2}$. Subjects were excluded if they had a history of respiratory, circulatory, or neurological disorders or if they had reduced chest and abdominal mobility, because this makes it difficult to measure the distances targeted by the BMMD. This study was approved

\section{QUICK LOOK}

\section{Current knowledge}

Chest and abdominal wall mobility is an important component of respiratory assessment. Loss of chest and abdominal wall mobility may result in respiratory complications. Chest and abdominal mobility assessments are not routine in clinical care.

\section{What this paper contributes to our knowledge}

Linear regression equations can provide adequate estimates of the 3-dimensional (3D) distances of markers on the chest and abdominal wall during deep breathing (DB). The results suggest that a novel breathing movement-measuring device (BMMD) is useful for quantitatively assessing DB movement of the chest and abdominal wall in healthy young males.

by our local ethics committee (10-195), and all subjects provided their informed consent.

\section{Breathing Movement-Measuring Device}

The BMMD (Pacific Medico Co, Tokyo, Japan), which is custom made, is a pen-sized mechanical device comprising a main body with a distance scale $(\mathrm{mm})$, an angle scale, a tension spring, a metal rod, a coupling part with a mark, and a discoid contact part with a ball joint (Fig. 1). A movement distance of the observation points during breathing could be measured from the position of the mark, which moves on the distance scale via the discoid contact part.

\section{Breathing Movement Scale (BMS)}

A BMS was developed to provide a more refined assessment of breathing movements, which can be used among all healthcare professionals. We developed a BMS that assessed QB and DB movements of the chest and abdominal wall based on a scale of -1 to 8 . We measured the breathing movements for 3 regions: upper chest (third rib), lower chest (eighth rib), and abdomen (midpoint between the xiphoid process and umbilicus). The upper and lower chest regions were also divided into right and left sides. Finally, 5 observation points were set for evaluation of breathing patterns, chest and abdominal mobility, synchrony and asymmetry of chest wall movements, and asynchrony of chest and abdominal wall movements. These points were chosen as representative sites, because they show greater movement of the upper chest, lower chest, and abdomen and reflect the characteristics of normal 


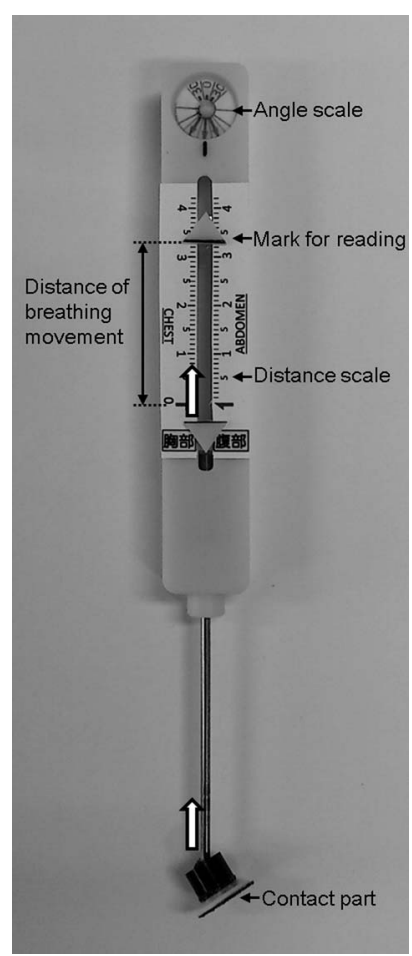

Fig. 1. The breathing movement-measuring device (BMMD) is a pen-sized mechanical device. Breathing movements of the chest and abdominal wall are measured from the position of the mark, which moves on the distance scale via the discoid contact part while maintaining the position of the BMMD at the measurement angle.

breathing. ${ }^{2}$ Breathing movement was defined as the 3D distance at the observation point from the end of an expiration to the end of an inspiration. The 3D distances of the observation points were measured while in the supine position because a sitting position caused compensatory movements. $^{2}$

In addition, the criteria for scaling QB movements and chest and abdominal mobility were specified on the basis of normal QB and DB movements. ${ }^{2}$ These criteria were as follows: the scale value of $-1,<0 \mathrm{~mm} ; 0,<10$ th percentile of normal QB; 1, 10th through 90th percentiles of QB; 2 and 3, an interval such that the distance between the 90th percentile of QB and the 10th percentile of normal DB was divided into halves; 4 , 10th through 25 th percentiles of DB; 5, 25th through 50th percentiles of DB; 6, 50th through 75th percentiles of DB; 7, 75th through 90th percentiles of DB; and $8,>90$ th percentile of DB. The scale values from -1 to 3 were applied to assess QB movement, and the scale value of 1 was considered normal. The scale value of -1 indicated paradoxical breathing. To assess DB movements, scale values of $0-8$ were applied, with the normal range on this scale being $4-7$.

\section{Breathing Movement Measurements}

Using a 3D motion analysis system (Vicon MX, Vicon Motion System, Oxford, United Kingdom), breathing movements were measured during DB while in the supine position. This system comprised 8 infrared cameras that tracked the movement trajectories of 14 -mm passive markers attached to the chest and abdominal wall. Camera images were sampled at a rate of $50 \mathrm{~Hz}$, and motion data were processed using Workstation 5.2.4 software (Vicon Motion System) to produce 3D coordinates for each marker. These coordinates were smoothed using a Woltring filter incorporated in Workstation 5.2.4 software (Vicon Motion System), and the distances between the markers were calculated using spreadsheet software (Excel, Microsoft, Redmond, Washington). A Woltring filter, which is equivalent to a double Butterworth filter, can process data with unequal sampling intervals and boundary conditions. Five passive markers were bilaterally placed along the vertical line through the medial one-third of the clavicle, third rib (upper chest), and eighth rib (lower chest) and along the vertical line through the umbilicus at the midpoint between the xiphoid process and umbilicus (abdomen). The range of movement was measured as the 3D distance during the inspiratory phase of the respiratory cycle. Based on a single breathing cycle, distances were calculated and expressed in millimeters.

\section{Procedure}

The subjects were asked to remove their shirts and loosen their pants while in the supine position. They were instructed not to talk or move during the measurements. Breathing was controlled and based on the distances targeted by the BMMD: $1 \mathrm{~cm}$, breathing condition $1 ; 2 \mathrm{~cm}$, breathing condition 2; and $3 \mathrm{~cm}$, breathing condition 3 . For healthy subjects, the maximal breathing amplitude was determined based on the average 3D distances of the chest and abdominal wall during DB (approximately $3 \mathrm{~cm}$ ). The values of the BMS corresponding to the breathing conditions were as follows: breathing condition 1 , breathing condition 2 , and breathing condition 3 were the $1 / 2$ scale (abdomen/chest), the 3/4 scale, and the 5/6 scale, respectively. The subjects were provided feedback information by the examiner for the distances targeted by the BMMD. The subjects were asked to maintain the end of an inspiration and expiration for at least $2 \mathrm{~s}$. The BMMD distances were measured and recorded in millimeters.

When assessing breathing movements, for the upper and lower chest, the BMMD was held in a $30^{\circ}$ cranially and slightly (approximately $10^{\circ}$ ) laterally inclined position from the vertical line. For the abdomen, the BMMD was held in the vertical position using a tripod. Determination of this position was based on the average angle between the ver- 


\section{Estimating Breathing Movements Using a New Device}

Table 1. 3D and BMMD Distances at Different Breathing Amplitudes

\begin{tabular}{|c|c|c|c|c|c|c|c|c|c|c|}
\hline & \multicolumn{3}{|c|}{ Breathing Condition 1} & \multicolumn{3}{|c|}{ Breathing Condition 2} & \multicolumn{3}{|c|}{ Breathing Condition 3} & \multirow[b]{2}{*}{$P($ Difference $) \S$} \\
\hline & $\begin{array}{c}3 \mathrm{D} \\
(\mathrm{mm})^{*}\end{array}$ & $\begin{array}{c}\text { BMMD } \\
(\mathrm{mm}) \dagger\end{array}$ & $\begin{array}{c}\text { Difference } \\
(\mathrm{mm}) \ddagger\end{array}$ & $\begin{array}{c}3 \mathrm{D} \\
(\mathrm{mm})^{*}\end{array}$ & $\begin{array}{l}\mathrm{BMMD} \\
(\mathrm{mm}) \dagger\end{array}$ & $\begin{array}{l}\text { Difference } \\
(\mathrm{mm}) \ddagger\end{array}$ & $\begin{array}{c}3 \mathrm{D} \\
(\mathrm{mm})^{*}\end{array}$ & $\begin{array}{c}\text { BMMD } \\
(\mathrm{mm}) \dagger\end{array}$ & $\begin{array}{l}\text { Difference } \\
(\mathrm{mm}) \ddagger\end{array}$ & \\
\hline \multicolumn{11}{|c|}{ Upper chest } \\
\hline Right & $14.8 \pm 1.7$ & $11.1 \pm 1.7 \|$ & $3.7 \pm 1.7$ & $25.6 \pm 1.4$ & $20.4 \pm 1.0 \|$ & $5.2 \pm 0.8$ & $35.9 \pm 1.9$ & $29.9 \pm 1.5 \|$ & $6.0 \pm 0.7$ & $\begin{array}{l}\mathrm{B} 1 \text { vs B2 }(P=.005) \\
\text { B1 vs B3 }(P<.001) \\
\text { B2 vs B3 }(P=.18) \\
\text { B1 vs B2 }(P=.02)\end{array}$ \\
\hline Left & $13.7 \pm 1.5$ & $10.9 \pm 1.1 \|$ & $2.8 \pm 1.2$ & $25.0 \pm 0.7$ & $20.2 \pm 0.9 \|$ & $4.8 \pm 1.3$ & $35.5 \pm 2.4$ & $29.8 \pm 1.3 \|$ & $5.7 \pm 1.6$ & $\begin{array}{l}\mathrm{B} 1 \text { vs B3 }(P<.001) \\
\mathrm{B} 2 \text { vs B3 }(P=.43) \\
\mathrm{B} 1 \text { vs B2 }(P=.11)\end{array}$ \\
\hline \multicolumn{11}{|c|}{ Lower chest } \\
\hline Right & $14.6 \pm 1.5$ & $10.6 \pm 1.0 \|$ & $4.0 \pm 1.5$ & $24.8 \pm 2.3$ & $19.8 \pm 1.8 \|$ & $5.0 \pm 1.2$ & $34.7 \pm 3.3$ & $29.2 \pm 2.7 \|$ & $5.5 \pm 1.2$ & $\begin{array}{l}\mathrm{B} 1 \text { vs B3 }(P=.01) \\
\mathrm{B} 2 \text { vs B3 }(P=.97) \\
\mathrm{B} 1 \text { vs B2 }(P=.23)\end{array}$ \\
\hline Left & $14.0 \pm 2.2$ & $10.1 \pm 0.6 \|$ & $3.9 \pm 2.0$ & $25.6 \pm 2.2$ & $20.5 \pm 1.2 \|$ & $5.1 \pm 2.0$ & $35.0 \pm 2.1$ & $29.5 \pm 1.8 \|$ & $5.5 \pm 2.5$ & $\begin{array}{l}\mathrm{B} 1 \text { vs B3 }(P=.06) \\
\mathrm{B} 2 \text { vs B3 }(P>.99) \\
\mathrm{B} 1 \text { vs B2 }(p=.004)\end{array}$ \\
\hline Abdomen & $14.2 \pm 2.0$ & $10.2 \pm 0.8 \|$ & $4.0 \pm 1.8$ & $27.5 \pm 1.5$ & $20.9 \pm 1.7 \|$ & $6.6 \pm 1.5$ & $35.0 \pm 2.7$ & $27.0 \pm 2.8 \|$ & $8.0 \pm 1.5$ & $\begin{array}{l}\text { B1 vs B3 }(P<.001) \\
\text { B2 vs B3 }(P=.13)\end{array}$ \\
\hline $\begin{array}{l}\text { Data are mean } \\
\text { * Distance me } \\
\dagger \text { Distance me } \\
\text { † Difference b } \\
\text { § Comparison } \\
\| \text { Comparison } \\
\text { B1-B3 = brea }\end{array}$ & $\begin{array}{l}\mathrm{SD}(n=10) \text {. } \\
\text { red by the 3-dim } \\
\text { red by the breath } \\
\text { een the } 3 \mathrm{D} \text { and } \\
\text { ween breathing } \\
\text { ween the } 3 \mathrm{D} \text { and } \\
\text { ng conditions } 1-3\end{array}$ & $\begin{array}{l}\text { ensional (3D) mot } \\
\text { ing movement-me } \\
\text { BMMD distances } \\
\text { onditions } \\
\text { BMMD distances } \\
\text { B }\end{array}$ & $\begin{array}{l}\text { tion analysis sy } \\
\text { easuring device } \\
\text { (3D distance - } \\
(P<.001) \text { TH }\end{array}$ & $\begin{array}{l}\text { em } \\
\text { BMMD) } \\
\text { BMMD distance) } \\
\text { BMMD distance }\end{array}$ & es (observed vi & were les & e 3D dista & & & \\
\hline
\end{tabular}

tical and straight lines passing through the starting and ending points of trajectories of inspiration movements in healthy subjects. At the end of expiration, the BMMD mark was adjusted to the starting point of the scale by lightly pressing the BMMD against the observation point. Breathing movements were calculated from this marked distance to the end of inspiration. At the same time, the 3D distances of the reflective markers on the observation points were measured.

\section{Statistical Analysis}

The sample size was determined using the results of a previous study ${ }^{15}$ that used this BMMD. The results revealed a minimal $r^{2}$ of 0.69 during QB (unpublished data). An adequate sample size was determined to be at least 9 subjects at $\alpha=0.05$ and $1-\beta=80 \%$. Results are presented as means $\pm \mathrm{SD}$. A paired $t$ test was used to compare the differences between the 3D and BMMD distances during the 3 breathing amplitudes. In addition, one-way repeated measures analysis of variance with a Bonferroni correction for multiple comparisons was used to assess the differences between the 3 breathing conditions in the differences between the 3D and BMMD distances. The relationship between the 3D distance (dependent variable) and
BMMD distance (independent variable) was assessed by linear regression with mixed effects, which was appropriate for repeated measurement of the same subject. The $\mathrm{r}^{2}$ and the standard error of the estimate (SEE) were calculated to assess the suitability of the regression equations and the accuracy of predictions. In addition, based on the obtained regression equations, the concordance rates of the BMS between the predicted scales from the BMMD distances and the actual scales from the 3D distances were calculated. Statistical analyses were performed using SPSS Statistics 19 (IBM, Armonk, New York) and R version 2.15.1 software (available at http://www.R-project.org; $a c$ cessed May 21, 2014). A $P$ value $<.05$ was considered significant.

\section{Results}

All of the measured BMMD distances were less than the 3D distances at each observation point (Table 1). The mean differences between the 3D and BMMD distances decreased according to the breathing amplitudes. Significant differences between breathing conditions 1 and 2, between breathing conditions 1 and 3 for the upper chest and abdomen, and between breathing conditions 1 and 3 for the right lower chest were found; however, no differ- 
Estimating Breathing Movements Using a New Device

Table 2. Estimating 3D Distances From BMMD Distances at Each Observational Point

\begin{tabular}{|c|c|c|c|c|c|c|}
\hline & $\begin{array}{c}\text { 3D } \\
\text { Distance } \\
(\mathrm{mm})\end{array}$ & $\begin{array}{l}\text { BMMD } \\
\text { Distance } \\
(\mathrm{mm})\end{array}$ & $\begin{array}{l}\text { Regression Equation } \\
\qquad(\mathrm{mm})\end{array}$ & $\mathrm{R}^{2}$ & SEE (mm) & $\begin{array}{c}\text { SEE/3D Distance } \\
(\%)\end{array}$ \\
\hline \multicolumn{7}{|c|}{ Upper chest } \\
\hline Right & $25.4 \pm 8.9$ & $20.5 \pm 7.9$ & $2.44+1.12 \times$ BMMD $^{*}$ & 0.99 & 1.2 & 4.7 \\
\hline Left & $24.7 \pm 9.2$ & $20.3 \pm 7.9$ & $1.49+1.14 \times \mathrm{BMMD}$ & 0.98 & 1.4 & 5.6 \\
\hline \multicolumn{7}{|c|}{ Lower chest } \\
\hline Right & $24.7 \pm 8.7$ & $19.9 \pm 8.0$ & $3.32+1.08 \times \mathrm{BMMD}$ & 0.98 & 1.3 & 5.0 \\
\hline Left & $24.8 \pm 9.0$ & $20.0 \pm 8.2$ & $3.13+1.09 \times \mathrm{BMMD}$ & 0.98 & 2.2 & 8.9 \\
\hline Abdomen & $25.6 \pm 9.0$ & $19.4 \pm 7.3$ & $1.97+1.22 \times \mathrm{BMMD}$ & 0.99 & 1.8 & 7.1 \\
\hline \multicolumn{7}{|c|}{$\begin{array}{l}\text { Distance data are means } \pm \mathrm{SD}(n=30) \text {. } \\
* \text { Distance measured using the breathing-movement measuring device (BMMD) } \\
3 \mathrm{D}=3 \text {-dimensional } \\
\mathrm{SEE}=\text { standard error of the estimate }\end{array}$} \\
\hline
\end{tabular}

Table 3. Breathing Movement Scale Corresponding to the 3D and Predicted 3D Distances in Different Breathing Amplitudes

\begin{tabular}{|c|c|c|c|c|c|c|c|c|c|}
\hline & \multicolumn{3}{|c|}{ Breathing Condition 1} & \multicolumn{3}{|c|}{ Breathing Condition 2} & \multicolumn{3}{|c|}{ Breathing Condition 3} \\
\hline & Scale* & Predicted Scale $\dagger$ & Concordance $\ddagger$ & Scale & Predicted Scale & Concordance & Scale & Predicted Scale & Concordance \\
\hline \multicolumn{10}{|c|}{ Upper chest } \\
\hline Right & $3(2-3)$ & $3(2-3)$ & $8 / 10$ & $5(4-5)$ & $5(4-5)$ & $10 / 10$ & $6(6-7)$ & $6(6-7)$ & $10 / 10$ \\
\hline Left & $3(3-3)$ & $3(3-3)$ & $10 / 10$ & $5(4-5)$ & $5(4-5)$ & $7 / 10$ & $6(6-7)$ & $6(6-7)$ & $9 / 10$ \\
\hline \multicolumn{10}{|c|}{ Lower chest } \\
\hline Right & $3(2-3)$ & $3(2-3)$ & $7 / 10$ & $5(4-5)$ & $5(4-5)$ & $9 / 10$ & $6(6-7)$ & $6(6-7)$ & $8 / 10$ \\
\hline Left & $3(2-3)$ & $3(2-3)$ & $8 / 10$ & $5(4-5)$ & $5(4-5)$ & $8 / 10$ & $6(6-7)$ & $6(6-7)$ & $8 / 10$ \\
\hline Abdomen & $1(1-2)$ & $1(1-2)$ & $6 / 10$ & $4(4-5)$ & $4(4-5)$ & $7 / 10$ & $5(5-6)$ & $6(4-6)$ & $8 / 10$ \\
\hline \multicolumn{10}{|c|}{$\begin{array}{l}\text { Data represent the median (range) }(n=10) \text {. } \\
\text { * Breathing movement scale corresponding to the 3-dimensional (3D) distance } \\
\dagger \text { Breathing movement scale corresponding to the 3D distance predicted from the } \\
\text { ¥ Concordance ratio of the breathing movement scale of the predicted 3D distan }\end{array}$} \\
\hline
\end{tabular}

ences were found for the left lower chest. The regression equations for estimating the 3D distances from the BMMD distances gave high $\mathrm{r}^{2}$ and low SEE values for all the observation points (Table 2). The SEE value for the left lower chest region was relatively high compared with that of the other observation points. The concordance rates of the BMS were from $6 / 10(60 \%)$ for the abdomen to $10 / 10$ (100\%) for the upper chest (Table 3 ); the average concordance rate was $123 / 150(82 \%)$.

\section{Discussion}

The results of this study demonstrated that, for each observation point, the derived regression equations estimated the 3D distances from the BMMD distances with an acceptable accuracy. Therefore, this BMMD may be useful for quantitatively assessing DB movements of the chest and abdominal wall in healthy young males.

The 5 observation points, which divided the chest and abdomen into 3 regions (upper chest, lower chest, and abdomen) and the chest into right and left sides, reflected the characteristics of breathing movement related to the effects of age, sex, and posture. ${ }^{2}$ In addition, measurements of chest excursion using a tape measure have been made at 3 similar levels (axillary, xiphisternal, and abdominal). ${ }^{14}$ Tape measurements are simple and inexpensive. However, this method is not suitable for evaluating any asymmetry or abnormality in the mobility of the chest and abdominal wall. The BMS using the BMMD has the advantage of assessing asymmetry and abnormality in mobility, because it is based on measurements of both sides of the chest and provides reference values. ${ }^{2}$

Breathing movement less than the scale value of 4 , in which the lower limit is the 10th percentile of the normal $\mathrm{DB}$, is considered an indication of limited chest and abdominal wall mobility. The older subject, who had less chest mobility than the younger subject, was included in the normal range (scale, $4-7) .^{2}$ Therefore, if BMS is less than the scale value of 4 , it is obviously regarded as an indication of limited chest and abdominal wall mobility. In 
addition, in the healthy subjects, chest and abdominal mobility have reportedly been symmetrical. ${ }^{12}$ In our previous study, ${ }^{2}$ there were no significant differences in the 3D distances of the DB movements between right and left sides. The maximum difference between right and left was $3.0 \mathrm{~mm}$, which was smaller than the minimum distance on the BMS scale. Therefore, if the difference between right and left side was $>1$ point on the scale, it was considered an indication of asymmetry in chest mobility.

For the markers at each observation point, all of the BMMD distances were less than the 3D distances. The reasons for these results were as follows. First, the trajectories of the markers on the chest and abdominal wall were not linear, although the BMMD distances were measured as linear distances. Second, the soft tissue at an observation point was compressed by the tension spring of the BMMD, which resulted in a weak force. For an individual with more subcutaneous fat and a deviated direction of breathing movement, the differences between the 3D and BMMD distances would be large. Third, the measuring angle of the BMMD was not necessarily in accordance with the direction of an individual's breathing movement.

The differences between the 3D and BMMD distances were associated with the breathing amplitudes used. These differences were significantly less for breathing condition 1 than for the other breathing conditions. This may result from decreases in compression caused by the tension spring in lower amplitudes. Therefore, to assess a movement distance during $\mathrm{QB}$, the regression equations derived in this study may not be appropriate for estimating the 3D distances of breathing movement.

The 3D distances of the observation points were estimated with acceptable accuracy. The SEE values were $<1.8 \mathrm{~mm}$ (7.1\% of the mean 3D distance), except for those for the left lower chest. SEE for the left lower chest was relatively large $(2.2 \mathrm{~mm} ; 8.9 \%$ of the mean 3D distance). These results were consistent with those of the previous studies, which showed relatively lower reliability and agreement. ${ }^{2,15,16}$ This may be explained by assuming that the differences between the 3D and BMMD distances were relatively more variable among the subjects, that is, variations in the trajectory of the left lower chest marker were relatively large.

The BMS assesses DB movements on the chest and abdominal wall on a scale of $0-8$. The minimal interval of this scale for DB was found to be approximately $3.5 \mathrm{~mm}$, which was calculated from the data for healthy subjects $20-74$ y of age. ${ }^{2}$ For almost all the observation points, the 95\% confidence intervals for the predicted values of the regression equations $(1.96 \times$ SEE) were less than this value, indicating that the measurement errors were within 1 unit of the BMS. In fact, measurement errors between the predicted and actual scales were within 1 point on the scale, and approximately $80 \%$ of the predicted scales agreed with the actual scale. For the abdomen, the concordance rate for breathing condition 1 was the lowest, although the 95\% CI for the left lower chest was more than this minimal interval. This is the reason why the $3 \mathrm{D}$ distances for breathing condition 1 on the abdomen were closer to its boundary value between scales compared with the other observation points and breathing conditions. Therefore, if the BMMD mark is located near the boundary value, careful BMMD measurements will be required.

Furthermore, since the amplitudes of the breathing condition 1 at the abdomen were comparable with the scale value of 1 , when the regression equation derived from the abdominal wall movement data during $\mathrm{QB}^{2}$ was applied to estimate the $3 \mathrm{D}$ distances, the concordance rate of the abdomen improved to $8 / 10$. Therefore, for estimating breathing movements of scale 1 , the regression equations derived from the data of the QB condition are appropriate.

Chest and abdominal wall mobility is one of the important components of a respiratory assessment and physical exercise. The present results demonstrate that the BMMD distances can estimate the 3D distances of the breathing movement of the chest and abdominal wall with acceptable accuracy, and suggest that the BMS using this BMMD may be useful for performing objective assessments of chest and abdominal wall mobility in healthy young males. Moreover, if the BMS is displayed on the BMMD, it may be more useful for assessing chest and abdominal mobility during a physical examination.

However, on the BMS, the measurement of 3D distances was performed while in the supine position only to avoid compensatory movements like spine movements, which have been shown to have different characteristics between the supine and sitting positions. ${ }^{2}$ Therefore, when measured in the sitting position, a 3D distance may not maintain acceptable accuracy for scaling chest and abdominal wall mobility. Furthermore, accuracy of predicting these 3D distances may be influenced by age, sex, disease (eg, spinal and thoracic deformity), body type, and the fixation condition used for the BMMD.

The results of this study were based on the data collected from healthy young males. Therefore, this study did not attempt to address the effects of age, sex, and disease on these measurements. Our previous study ${ }^{2}$ showed that there was a significant negative relationship between age and $3 \mathrm{D}$ distances in the $\mathrm{DB}$ movement, although there is no significant relationship between the 3D distances and sex. However, the breathing movement directions had no significant difference between age and between sex. Therefore, it is believed that the regression equations in this study may be applicable to another healthy person, but not to patients with spinal and thoracic deformities who have abnormal breathing movement. It is necessary to find an- 


\section{Estimating Breathing Movements Using a New Device}

other measuring angle to attain acceptably accurate measurements for such patients.

The results of this study were derived from subjects with normal body types and fixation using a tripod. Thus, when there is more subcutaneous fat at an observation point, the 3D distances may be underestimated by the BMMD. In this case, this effect may be decreased by placing extra padding such as a thin rubber sheet on the observation point to diminish the pressure from the tension spring indenting the body surface.

In addition, a previous study showed that good reliability between the 3D and BMMD distances and low values for the limits of agreement (chest, $<2.0 \mathrm{~mm}$; abdomen, $3.0 \mathrm{~mm}$ ) in a Bland-Altman analysis during QB were obtained by fixing the BMMD using a hand. ${ }^{15}$ For DB, if the wrist and elbow are fixed firmly, good reliability of the BMMD measurement can be maintained for patients with decreased chest and abdominal mobility. Furthermore, when the measuring angle of the BMMD and the direction of the breathing movement are not clearly matched, the mark of the BMMD cannot move smoothly. In this case, for more precise assessments, the measuring angle should be adjusted to a proper direction such that it moves smoothly, and the measuring angle can be determined from the angular scale of the BMMD. The auxiliary lines of a $15^{\circ}$ unit are displayed on the angle scale.

However, we did not investigate the effects of different age, sex, body types, fixation methods, or disease during the BMMD measurements. Therefore, additional studies are required to investigate the effects of these factors on BMMD measurements.

\section{Conclusions}

This study investigated the usefulness of a newly developed BMMD for estimating the 3D distances on the chest and abdominal wall during DB. The regression equations for estimating the 3D distances from the BMMD distances gave high $\mathrm{r}^{2}$ and low SEE values for all the observation points. The BMMD may be useful for quantitatively assessing breathing movements of the chest and abdominal wall in healthy young males.

\section{REFERENCES}

1. Verschakelen JA, Demedts MG. Normal thoracoabdominal motions. Influence of sex, age, posture, and breath size. Am J Respir Crit Care Med 1995;151(2 Pt 1):399-405.

2. Kaneko H, Horie J. Breathing movements of the chest and abdominal wall in healthy subjects. Respir Care 2012;57(9):1442-1451.

3. Leong JC, Lu WW, Luk KD, Karlberg EM. Kinematics of the chest cage and spine during breathing in healthy individuals and in patients with adolescent idiopathic scoliosis. Spine 1999;24(13):1310-1315.

4. Chu WC, Li AM, Ng BK, Chan DF, Lam TP, Lam WW, Cheng JC. Dynamic magnetic resonance imaging in assessing lung volumes, chest wall, and diaphragm motions in adolescent idiopathic scoliosis versus normal controls. Spine 2006;31(19):2243-2249.

5. Dureuil B, Cantineau JP, Desmonts JM. Effects of upper or lower abdominal surgery on diaphragmatic function. Br J Anaesth 1987; 59(10):1230-1235

6. Kristjánsdóttir A, Ragnarsdóttir M, Hannesson P, Beck HJ, Torfason B. Respiratory movements are altered three months and one year following cardiac surgery. Scand Cardiovasc J 2004;38(2):98-103.

7. Romagnoli I, Gigliotti F, Galarducci A, Lanini B, Bianchi R, Cammelli D, Scano G. Chest wall kinematics and respiratory muscle action in ankylosing spondylitis patients. Eur Respir J 2004;24(3): 453-460.

8. Donath J, Miller A. Restrictive chest wall disorders. Semin Respir Crit Care Med 2009;30(3):275-292.

9. Sharp JT, Goldberg NB, Druz WS, Danon J. Relative contributions of rib cage and abdomen to breathing in normal subjects. J Appl Physiol 1975;39(4):608-618.

10. Gilbert R, Auchincloss JH Jr, Peppi D. Relationship of rib cage and abdomen motion to diaphragm function during quiet breathing. Chest 1981;80(5):607-612.

11. Cala SJ, Kenyon CM, Ferrigno G, Carnevali P, Aliverti A, Pedotti A et al. Chest wall and lung volume estimation by optical reflectance motion analysis. J Appl Physiol 1996;81(6):2680-2689.

12. Ragnarsdóttir M, Kristinsdóttir EK. Breathing movements and breathing patterns among healthy men and women 20-69 years of age. Reference values. Respiration 2006;73(1):48-54.

13. Bockenhauer SE, Chen H, Julliard KN, Weedon J. Measuring thoracic excursion: reliability of the cloth tape measure technique. J Am Osteopath Assoc 2007;107(5):191-196.

14. Malaguti C, Rondelli RR, de Souza LM, Domingues M, Dal Corso S. Reliability of chest wall mobility and its correlation with pulmonary function in patients with chronic obstructive pulmonary disease. Respir Care 2009;54(12):1703-1711.

15. Kaneko H. Assessing the reliability and validity of a newly developed breathing movement measuring device. J Phys Ther Sci 2013; 25(4):425-429.

16. Olsén MF, Romberg K. Reliability of the Respiratory Movement Measuring Instrument, RMMI. Clin Physiol Funct Imaging 2010; 30(5):349-353. 